\title{
Asymmetrical Genesis by Remanufacture of Antielectrons
}

\author{
Dirk J. Pons ${ }^{1}$, Arion D. Pons ${ }^{2}$, Aiden J. Pons ${ }^{3}$ \\ ${ }^{1}$ Department of Mechanical Engineering, University of Canterbury, Christchurch, New Zealand \\ ${ }^{2}$ University of Canterbury, Christchurch, New Zealand \\ ${ }^{3}$ Rangiora New Life School, Rangiora, New Zealand \\ Email: "dirk.pons@canterbury.ac.nz, arion@pons.ws, aiden@pons.ws
}

Received 28 September 2014; revised 23 October 2014; accepted 16 November 2014

Copyright (C) 2014 by authors and Scientific Research Publishing Inc.

This work is licensed under the Creative Commons Attribution International License (CC BY).

http://creativecommons.org/licenses/by/4.0/

(c) (i) Open Access

\section{Abstract}

Problem: The asymmetrical genesis problem concerns why the universe should have an abundance of matter over antimatter. Purpose: This paper shows how the baryogenesis and leptogenesis asymmetries may both be resolved. Approach: Design methods were used to develop a conceptual mechanics for the remanufacturing processes that transform particles in the decay processes. This was based on the structures for the photon, electron, antielectron, proton and antineutrino as previously identified as logical necessities for the beta decay process, and represented as a non-local hidden-variable design with discrete fields. Findings: The solution is given in terms of a mechanics that defines the transformation of discrete field structures in particles. The genesis problem is shown to be solvable. The mechanics describes pair production of an electron and antielectron from two initial photons, and subsequent remanufacture of the antielectron into a proton. It is predicted that two antineutrinos would be emitted, which are testable and falsifiable. The theory identifies that the role of the antineutrinos is to remove the antimatter handed field structures. The original electron and proton may bond to form a simple hydrogen atom, or combine via electron capture to form a neutron and hence heavier nuclides. The subsequent preponderance of the matter pathways in the genesis production sequence is also addressed and is explained as domain warfare between the matter and antimatter species. Originality: The concept of remanufacture of antielectrons into protons, with emission of antineutrinos, is novel. Extensions of the theory explain the nuclides. Consequently the theory explains from pair production up to nuclear structure, which is also original.

\section{Keywords}

Asymmetry, Antimatter, Annihilation, Chirality, Proton Decay, Non-Local Hidden-Variable, NLHV 


\section{Introduction}

\subsection{Background}

A deep foundational question is why the universe should have an abundance of matter over antimatter. This is the asymmetrical baryogenesis problem. The energy at genesis should have created equal amounts of matter and antimatter, which should be subsequently annihilated. While it is not impossible that there might be parts of the universe that consist of antimatter, and thereby balance the matter, neither is there any evidence that this is the case [1] [2]. Therefore it is generally accepted that the observed matter universe is more likely a result of an asymmetrical production of matter in the first place. But what might have caused matter to predominate? Charge conservation, which applies everywhere else in physics and is generally thought to apply to the universe as a whole, requires two processes: one to create a predominance of protons over antiprotons (baryogenesis), and another to make electrons rather than positrons (leptogenesis). So two processes are required and both are obscure. The Sakharov criteria for the imbalance of matter-antimatter require, inter alia, that charge-parity (CP symmetry) violation must occur [3]. However the mechanism for CP violation is uncertain. In this paper we provide a novel alternative solution to both the baryogenisis and leptogenesis problems. Somewhat unusually, the solution does not require any new particles. It only uses known particles, but it does require them to have a specific covert-structure, reminiscent of a type of non-local hidden-variable (NLHV) design, which we elaborate.

\subsection{Existing Approaches to the Asymmetry}

At present neither the Standard Model, quantum mechanics (QM), nor supersymmetry, can satisfactorily explain baryogenesis [4]. More complex models of those theories may yet be successful, or it may be that a different physics is required. Existing theories include the idea that the initial conditions imposed on the universe favoured matter. In other words the constraint came from outside the universe. This explanation is generally dismissed as unnatural [2]. Another idea, also not considered likely is that the antimatter is still around [5], in some hidden places in the universe. Many of the theories attempt to directly show how CP or CPT violation may generate asymmetry [6] [7]. Leptogenesis is a common focus area, e.g. via gravity waves [8] or via neutrino oscillation and the see-saw mechanism [9]. There is also leptogenesis using a hypothesised singlet neutrino that subsequently decays preferentially into antineutrinos, which are in turn converted to matter. Alternatively, that neutrinos and antineutrinos have slightly different native properties [10]. Sterile neutrinos are also a contender [11] [12]. There are theories that propose electroweak baryogenesis in the Standard Model [13]-[15]. Modifications to the Standard Model have also been proposed. One pathway is that right-handed neutrinos might decay into leptons, and those in turn converted by sphalerons into bosons [16]. The sphalerons are assumed to have existed at the high temperatures at the formation of the universe, and not thereafter. However right-handed neutrinos are controversial as they have not yet been observed, and even the existence of mass for standard neutrinos is uncertain. A variety of supersymmetry theories exist including grand unification theories (GUT), the Affleck-Dine mechanism [17], and heavy Majorana neutrinos [18]-[20]. However, the evidence for supersymmetry is not compelling, and the simpler versions are not evident in the LHC data from CERN [21] as might be expected. This is not a complete list, but rather indicative of the theoretical approaches. There are many hybrids between these approaches, and some also address dark matter [22]-[25]. There is no obvious way to judge the validity of the many candidate solutions.

A characteristic of many of these theories is that they are particle-centric: they formulate the problem in terms of a new particle being required to carry each new interaction. This approach has several difficulties. It is not easy to empirically validate the existence of the particles. Smaller particles require the construction of higher energy, and hence larger, colliders. Even with existing colliders, the empirical evidence for certain particles, especially those of super-symmetry, is lacking where it might be expected to be evident. And the unreactivity of the neutrino particles, which feature in many of the theories, makes them intrinsically difficult to observe. Also, there is no single strong candidate solution to have emerged from the particle perspective, only many contenders. So there is no obvious ontological convergence towards a solution, other than a predominance of neutrino-based solutions.

\subsection{Purpose}

Recent developments have suggested that the physics at the next deeper foundational level can be described by 
permitting particles to have internal functionality including the emission of discrete fields. A specific solution is the Cordus theory [26], which has shown wide-ranging relevance. The theory is a deeper interpretation than QM. It recovers QM and explains how the probabilistic formalism of QM arises from a deeper deterministic mechanics. Thus it proposes that physical realism exists and underpins the functional behaviour observed at the fundamental level. This is consistent with the original EPR idea [27]. The theory is therefore broadly compatible with $\mathrm{QM}$, likewise also with general relativity and string/M theory.

The purpose of this paper was to use this new foundational physics to attempt a solution to the problem of asymmetrical genesis of matter. This is worth attempting for several reasons. First, that existing theories have fared poorly and it is therefore worth looking more widely for solutions. Second, the Cordus theory has separately shown the feasibility of explaining the table of nuclides (stability behaviour $\mathrm{H}$ to $\mathrm{Ne}$ ) [28] from first principles of the strong force [29], which has not been achieved by other theories. There would be great value in also being able to explain the asymmetrical genesis process, since this would give a comprehensive production sequence for matter.

\section{Method}

\subsection{Approach}

This new theory purports to provide a solution based on physical realism, using finer-scale (covert) structures. This is similar to a hidden variable solution with discrete fields. Any concept based on hidden-variables will be challenged, and therefore a brief justification is provided as to why this approach should be considered scientifically sound. It is generally known that the Bell type inequalities [30]-[32] preclude local hidden-variable solutions. They do not preclude non-local hidden-variable (NLHV) solutions [33] [34]: this is not contentious. However the non-local sector has not been productive, since it has been difficult to find candidate solutions in this area. The only historical candidate of note is the de-Broglie-Bohm theory of the pilot-wave [35] [36], but this is still limited in its application to particle-locus situations, and has poor relevance to wider physics such as the nucleosynthesis situation under examination here. It is notable that the other theories of fundamental physics also have their equivalents of "hidden" variables. Thus quantum mechanics has its "intrinsic" variables for which it is unable to provide any deeper explanation. Similarly string/M theory has its extra "dimensions" which are presumed to be hidden, but also unexplained. So there cannot be any objection on philosophical grounds of implausibility to the idea of covert variables, since all theories have them.

Superficially, the Cordus theory might appear to be contrary to quantum mechanics. Actually that is unfounded, since the theory accommodates QM. It merely conceptualises a deeper physical mechanics where the stochastic nature of QM emerges at a coarser level. The conjectural starting point of the Cordus theory is speculative, but that hardly makes it inadmissible. It is true that the theory is unorthodox, but all theories have their speculative foundations, especially when they are young. The Cordus theory makes its starting premises explicit, and documents its assumptions in a series of lemmas. It achieves a strong internal logical consistency.

A further justification for seeking solutions wherever they may be found is the theoretical work showing that quantum mechanics is incapable of any further refinement [37], and hence will be unable to represent any new physics that may exist.

\subsection{Method}

The Cordus theory [26] proposes that particles have a finer-scale structure comprising internal structures and discrete fields. It has identified the general principles for these structures and predicted specific designs for each of the particles in the Standard Model and the corresponding antimatter species. These structures were identified using a design methodology, on the basis of identifying what minimal set of physical structures would be necessary and sufficient to explain the observed phenomena of wave-particle duality. Thus the initial concept was designed on the principle of requisite variability.

A systems engineering design method was used for the overall theoretical development. The systems part of the method ensures that the sub-theories are logically consistent with each other, and the design part involves taking the functional requirements (observed physics) and inferring the requisite attributes (internal mechanics of the phenomenon).

The discrete field model of the Cordus theory, specifically its HED mechanics (explained below) [38] was 
used to explore the theoretical feasibility of various genesis production routes. Several candidate processes were examined, but only one was tractable and is reported here. This involves the transformation of the antielectron (positron) into the proton. The following sections elaborate by showing the overall genesis process.

\section{Results}

\subsection{Covert Structures of the Cordus Theory}

The core postulate of the Cordus theory is that all particles are one dimensional structures of finite length, and from their two ends emit discrete forces that travel down flux lines in three orthogonal directions (hence hyperfine emission directions, or HEDs). This combination of internal structure and external discrete field structures is called a particule. A larger theory has been developed by systematic and logical development of the implications of the conjectured structures. This has been used to explain wave-particle duality, unification, nuclides (H to Ne), and time [26] [28] [29] [39]. This has demonstrated that the initial idea of covert structures, which was developed to explain wave-particle duality, does have the capability to explain a wide range of phenomena with otherwise problematic causality, i.e. the theory has internal and external construct validity. This Cordus theory is the starting point for the present work. The results show that it is indeed possible to conceive of a solution to the genesis problem, with a surprising outcome that is falsifiable and could be testable.

\subsection{Pair Production at the Start of the Genesis Process}

The asymmetry problem is part of a larger genesis process. It is generally accepted that the genesis process starts with pair-production converting a photon pair into an electron and antielectron. Separate work has shown that the Cordus theory is able to describe how pair-production occurs at the fundamental level [40]. This also requires a differentiation of the matter-antimatter species, which is explained in terms of the handedness of emission of discrete forces [41]. The processes of annihilation have also been sketched out [42]. Thus a descriptive explanation of all the processes underpinning mass-energy equivalence is provided within the Cordus theory.

The input and outputs of the pair-production process is summarised in Figure 1, which also illustrates the proposed covert structures for these particules. The detailed processes are described elsewhere [40].

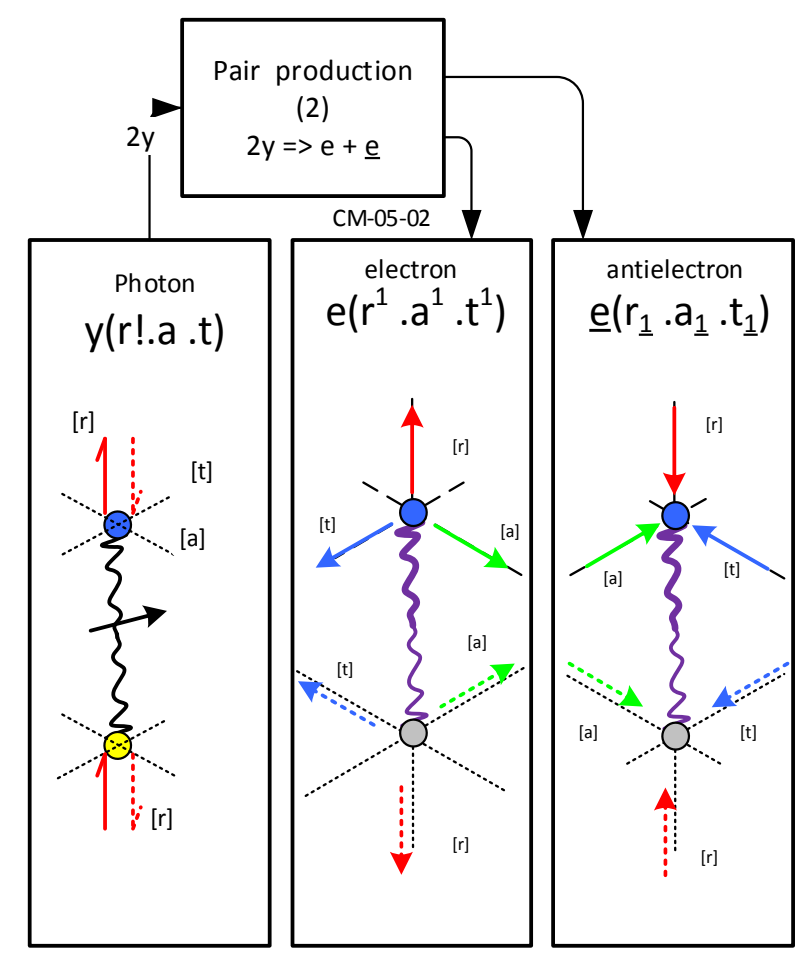

Figure 1. Pair production. Structures of the photon, electron, and antielectron particules according to the Cordus theory. 
The transformation from one type of particule to another, evident in the pair-production process, involves the reconfiguration of the patterns of discrete forces. These processes are described by a HED mechanics.

\subsection{Mathematical Formalism of the HED Mechanics}

The Cordus theory is mostly a conceptual theory, nonetheless a formalism exists for representing the discrete force arrangements of particules and predicting how those discrete forces may be remanufactured to create different particules. This is termed the HED mechanics.

The HED mechanics is a set of rules for the manipulation of discrete forces. These rules arise naturally from a core principle that a particule is defined by the pattern of discrete forces it emits, and therefore changes to the discrete forces cause the particule to change its nature. The pattern of discrete forces is represented in HED notation, which simply indicates the number of discrete forces in each of three orthogonal spatial directions $[r, a, t]$, their charge (negative: $x^{1}$, positive: $x_{1}$ ) and matter-antimatter hand (antimatter uses underscore, e.g. $X_{1}$ ). There are a number of assumptions in the HED mechanics, which are noted as lemmas. None are unreasonable, since they correspond to conservation principles that are already accepted in other physical theories. They are summarised as follows, as per [38].

1) The HED mechanics require the discrete forces to be conserved, rearranged, or even transformed, during transmutation and decay processes. Thus all discrete forces have to be accounted for, though they can be changed into other types as the annihilation theory shows.

2) The HED mechanics allow a charge- and hand-neutral complex of discrete forces to be added to any particule. This neutral complex comprises $x_{1.1}^{1.1}$ where $x$ is one of the HED axes. The complex is represented symbolically by $\uparrow \downarrow$ where $\uparrow=x_{1}{ }^{1}$ and $\downarrow=x_{1}{ }^{1}$. Being charge- and hand-neutral, this complex has no net energy. It is analogous to QM's idea of a vacuum fluctuation. Note that neither a single discrete force (say) $x^{1}$ nor a single pair (say) $\uparrow$ may be added to a particule ex vacuua: all such additions must be neutral as regards both charge and hand.

3) The structure $\uparrow \uparrow \uparrow=\left[r_{1}{ }^{1} \cdot a_{1}{ }^{1} \cdot t_{1}{ }^{1}\right]$ corresponds to a pair of photons, alternatively an electron-antielectron pair [42]. This set of discrete forces may be added to a particule as part of energy absorption.

The application of HED mechanics to a particule, or assembly of particules, is best understood as a remanufacturing process. The discrete forces are permitted to change to other axes (HEDs), and separate/combine into other groupings, and thereby redefine the identity of the particule. The HED mechanics does for this theory what Feynman diagrams do for 0-D points, the main difference being that the latter does not track the discrete fields.

\subsection{Re-Framing the Genesis Problem}

In the conventional narrative of physics, the asymmetrical baryogenesis problem is formulated as a need to bias the pair production process into the electron branch as opposed to the antielectron. However, here we make a conceptual departure by reformulating the problem as the need to dispose of the un-wanted matter-antimatter species after it is created, and remanufacture the antielectron into something different. If the anti-matter hand of the antielectron can be stripped out, then a matter-dominated universe would arise. The question is how the antimatter hand might be removed, and what the antielectron might change into.

\subsection{Neutrino Species}

A core proposition of the Cordus theory is that matter and antimatter are differentiated by hand, and that hand corresponds to the sequence of emission of the discrete forces [41]. For 3-D space there are only two ways this sequence can be arranged, hence only two species. Related to this, the Cordus decay theory [38] identified that the neutrino species are agents for changing the handedness of an assembled particule, while preserving charge. In other words, the neutrino species carry away unwanted matter or antimatter hand structures. In this theory the neutrino species are both a consequence and cause of species transformation. This explains why neutrino species are involved wherever there is a transformation between matter and antimatter products, as typically evident in the beta decays [38]. The covert structures of the neutrino and antineutrino are shown in Figure 2.

The Cordus theory also proposes that any particule is defined by its discrete force arrangements. This means that if some process rearranges the discrete forces, then that also changes the identity of the particule. Again, the 


\section{Neutrino $v$}

The neutrino is neutral since it has equal positive and negative charged discrete forces. The arrangement of those discrete forces is different to that of the neutron.

The HED energisation sequence is expected to create a corresponding spin angular momentum, the direction of which depends on the hand. Hence left-spin-hand arises from dexter hand energisation sequence.

Motion arises in the [a] directions as the particule lacks its own discrete forces in these axes. Where the fabric exists it uses discrete forces from the fabric, and then propagates at the speed of light as that is the saturated speed of the medium.

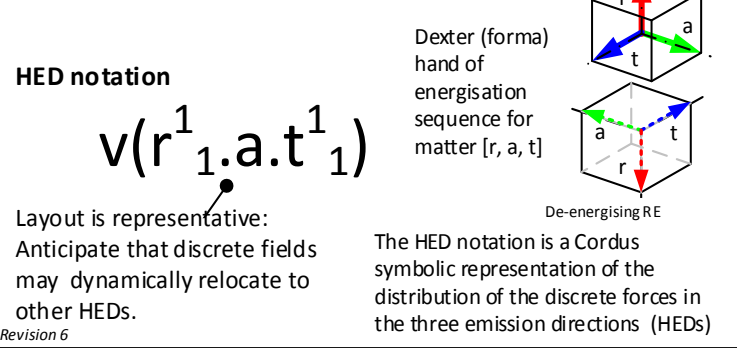

\section{Antineutrino $\underline{\mathbf{v}}$}

The antineutrino has the same discrete force count as the neutrino, but the opposite hand of energisation sequence. The unique spin directions of the neutrino and antineutrino arise due to the hand differences.

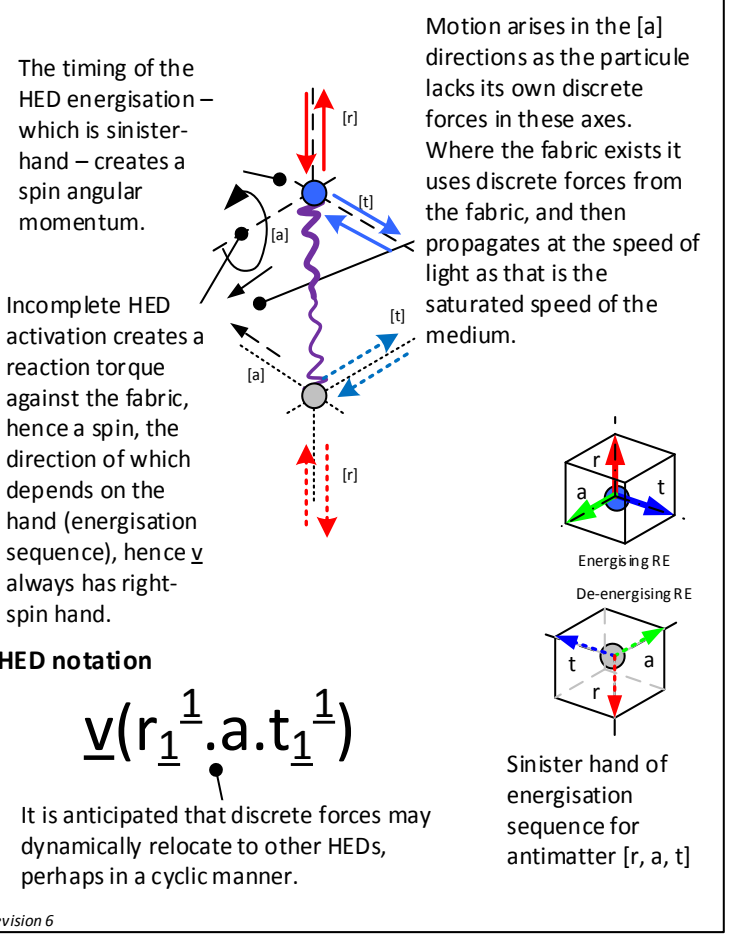

Figure 2. Neutrino species. These are the proposed structures of the neutrino and antineutrino. The imbalanced discrete forces are proposed as the cause for motion of these particules. The species are differentiated by hand, which is the energisation sequence of discrete forces. This also determines the direction of spin. Hence spin is a property of the species, rather than being an independent variable. Figure adapted from [38], by permission.

beta decays are a typical example, e.g. where the proton is converted into a neutron. It is also possible to convert one type of discrete force into another, as is shown in the Cordus theory for pair production [40] and annihilation [42]. These transformation processes and their underlying mechanics are represented in the HED mechanics. Since this theory defines the matter-antimatter species in terms of hand, any transformation that involves crossing from one species to the other is termed remanufacturing (L. manus, hand).

\subsection{Remanufacture of the Antielectron}

Now, the asymmetrical genesis problem has been rephrased as a need to change the species hand of the antielectron, and the neutrino has been identified as having the ability to remove handedness. Is it possible that the neutrino species are involved in remanufacturing the antielectron? If so, what method is there to predict how such a process works, and what its by-products would be? The HED mechanics answers these questions, and identifies a specific route whereby the antielectron may convert into a proton. The HED mechanics makes the specific prediction that the waste antimatter hand is discarded in two output antineutrinos. This is significant: being able to predict the process a priori and with a high-degree of specificity cannot be done with QM or Feynman diagrams.

A number of options were explored using the HED mechanics. At this point we have discovered only one production route that solves the genesis problem. The explanation follows.

Start with pair production, where the electron $(e)$ and antielectron $(\underline{e})$ pair arise from photons $(y)[40]$ :

$$
2 y \Rightarrow e\left(r^{1} \cdot a^{1} \cdot t^{1}\right)+\underline{e}\left(r_{\underline{1}} \cdot a_{\underline{1}} \cdot t_{\underline{1}}\right)
$$


Note the use of underscore to denote antimatter species, superscript for negative charge, and subscript for positive. Now add discrete forces: the energy equivalent of an additional two photons in the form of another electron-antielectron bolus $\left(\downarrow \downarrow \downarrow=r_{1}^{\underline{1}} \cdot a_{1}^{\underline{1}} \cdot t_{1}^{\underline{1}}\right)$. Also add a twin-pair $z=[\uparrow \downarrow]=\left(x_{1}^{1} \frac{1}{1}\right)$ which may be a vacuum fluctuation effect or photons (the theory is not specific on this point). These structures are justified in prior work [38]. Then:

$$
2 y+2 y+z \Rightarrow e\left(r^{1} \cdot a^{1} \cdot t^{1}\right)+\underline{e}\left(r_{\underline{1}} \cdot a_{\underline{1}} \cdot t_{\underline{1}}\right)+[\downarrow \downarrow \downarrow]+[\uparrow \downarrow]
$$

Now bring the discrete force pairs (arrows) into the antielectron and expand them to create a transitional structure $O$ :

$$
\begin{aligned}
& 4 y+z \Rightarrow e\left(r^{1} \cdot a^{1} \cdot t^{1}\right)+\underline{e}\left(r_{\underline{1}} \uparrow \downarrow \downarrow \cdot a_{\underline{1}} \downarrow \cdot t_{\underline{1}} \downarrow\right) \\
& 4 y+z \Leftrightarrow \underline{e}\left(r_{\underline{1}} \cdot a_{\underline{1}} \cdot t_{\underline{1}}\right)+O\left(r_{\underline{1} .11} \underline{1}^{1} \underline{1} \cdot a_{\underline{1} .1} \cdot \underline{t}_{\underline{1} .1}{ }^{1}\right)
\end{aligned}
$$

Note the branching assumption that it is the antielectron that transforms, not the electron-we explain why later. Intermediate structures like this are unstable since they have discrete forces of mixed hand (matter-antimatter) and are unbalanced, other examples being the $W$ and $Z$ bosons [38]. The synchronous interaction (strong force) [29] constrains them to reorganise into simpler and more stable structures. Extract the explicit structure of a proton $p\left(r_{11}{ }^{1} \cdot a_{1} \cdot t_{1}\right)$ [38] and put the remaining discrete forces into another transitional structure $O_{1}$ :

$$
4 y+z \Leftrightarrow e\left(r^{1} \cdot a^{1} \cdot t^{1}\right)+p\left(r_{11}{ }^{1} \cdot a_{1} \cdot t_{1}\right)+O_{1}\left(r_{1} \cdot \underline{1}^{1 \cdot 1} \cdot a_{\underline{1}}{ }^{\underline{1}} \cdot t_{\underline{1}}{ }^{\underline{1}}\right)
$$

By observation of the antineutrino structure $\underline{v}\left(r_{\underline{1}} \cdot a \cdot t_{\underline{1}}{ }^{1}\right)$ [38], note that the transitional structure can be uniquely partitioned into two antineutrinos:

$$
4 y+z \Rightarrow e\left(r^{1} \cdot a^{1} \cdot t^{1}\right)+p\left(r_{11}{ }^{1} \cdot a_{1} \cdot t_{1}\right)+\underline{v}\left(r_{\underline{1}}{ }^{1} \cdot a \cdot t_{\underline{1}}{ }^{1}\right)+\underline{v}\left(r_{\underline{1}}{ }^{1} \cdot a_{\underline{1}}{ }^{1} \cdot t\right)
$$

However this is not quite done, because other work [38] shows that the proton likely also has an implicit structure comprising balanced discrete forces that contribute to mass but not to change. Thus the full proton structure is expected to be:

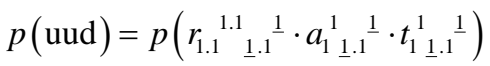

$$
\begin{aligned}
& =p\left[\left(r_{1.1}{ }^{1} \cdot a_{1} \cdot t_{1}\right)+\left(r_{\underline{1} \cdot 1^{1}}^{1} \cdot a_{\underline{1} \cdot 1^{1}}^{1} \cdot t_{\underline{1} \cdot 1^{1}}{ }^{\underline{1}}\right)\right] \\
& =p\left[\left(r_{1.1}{ }^{1} \cdot a_{1} \cdot t_{1}\right)+(r \uparrow \downarrow \cdot a \uparrow \downarrow \cdot t \uparrow \downarrow)\right] \\
& =p\left(r_{1.1}{ }^{1} \cdot a_{1} \cdot t_{1}\right)^{*} \text {. }
\end{aligned}
$$

Note that the implicit part is $[\uparrow \uparrow \uparrow]+[\downarrow \downarrow \downarrow]$, which by HED mechanics is four photons. These will need to be added to the initial inputs at Equation (2). Consequently the process of remanufacturing of the antielectron as a whole is predicted to be:

$$
8 y+z \Rightarrow e+p+2 v
$$

To sum up, the Cordus theory for genesis proposes that eight photons (possibly nine depending on the identity of the $z$ ) are remanufactured into an electron, a proton, and two antineutrinos. This prediction may be testable and falsifiable. The overall process, including the initial pair production, is shown in Figure 3.

However, there is still a question that must be addressed, which is why the production processes were biased to remanufacture the antielectron rather than the electron.

\subsection{Dominance of the Matter-Production Stream}

\subsubsection{Why Did the Matter Hand Prevail?}

This theory starts with the production of an electron-antielectron pair, after which the antielectron is remanufactured. By why the antielectron? Why were electrons not remanufactured to antiprotons, as in $n \times y=>\underline{e}+\underline{p}+2 v$ 


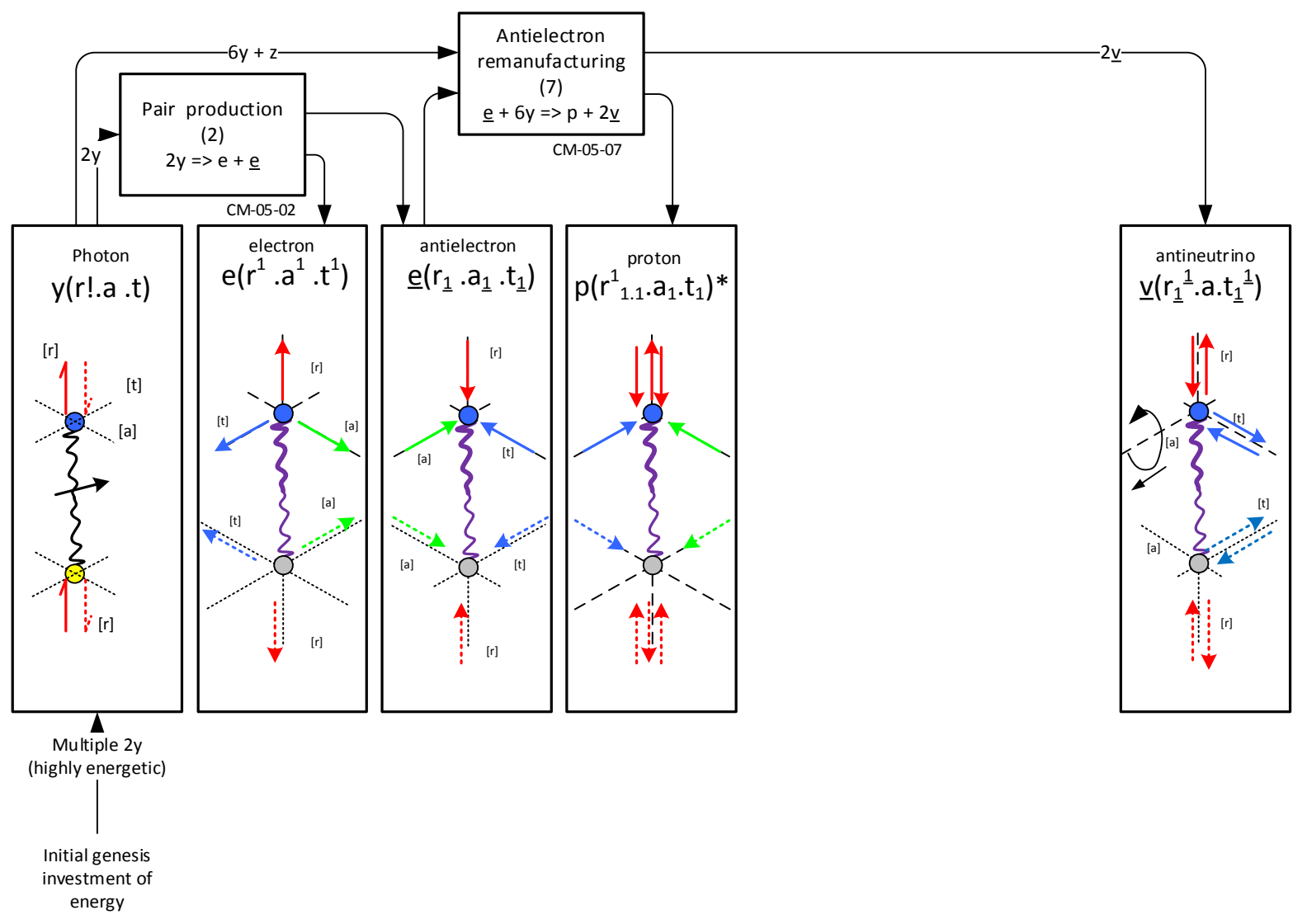

Figure 3. Asymmetrical genesis production stream. The HED mechanics predicts a process whereby the antielectron from pair production is remanufactured into a proton, with two antineutrinos ejected in the waste stream.

instead? While the HED mechanics solves the problems of how the asymmetry arises, and where the antimatter has gone to, there is a deeper question: what switched the production process to the matter route? We anticipate this may be answered in terms of warfare between the matter-antimatter species. Under this scenario, both production processes were initially at work. We imagine an initial extraordinarily energetic photon-pair colliding and producing an electron and antielectron, which then radiated further energetic photons. However, these photons would not have been able to propagate away, since there was no fabric [43] within which to move, so they would have been available for further pair-production sequences. The fabric comprises the discrete forces of all the other particules in the accessible universe, and is the NLHV equivalent of general relativity's spacetime.

With both streams of the remanufacturing process active, electrons and protons would have been created, alongside antielectrons and antiprotons. Any mixing across the species would have further annihilated back to photons. Those photons in turn were available to feed back into the production processes again, providing they were still energetic enough. Once some matter and antimatter particules had formed they would produce handed discrete forces and propagate those out, producing a fabric [43]. That fabric would carry a hand depending on its origin with matter or antimatter. In turn that fabric would bias the production processes it encountered to switch to the same hand. This is because particules, as they are created, must emit discrete forces into the fabric, and the fabric pressure pushes back and affects the emission. This also relates to the Cordus explanation for time dilation [39]. The massy particules would have extraordinary energy, hence high frequency, which would create an enormously high mass and strong fields.

In this scenario, domains of matter and antimatter formed, being multiple separate volumes of space where one of the hands dominated. Generally we would expect that these domains would be geometrically symmetrical with respect to each other. There would have been a stage of domain warfare as the domains aggregated, broke up, and forcibly converted parts of opposing domains. Perhaps the geometric symmetry broke down, so that the matter and antimatter domains were not the exact mirror images of each other. There are several possibilities for 
how the geometric asymmetry might arise: external perturbation from outside the universe; a random event in an increasingly large and disorderly system, i.e. a consequence of growing complexity; a natural oscillating dominance between the two species that was frozen in as the system expanded and cooled, i.e. the proto-universe was flipping between matter and antimatter dominated states initially. This last idea of frozen domain warfare is our currently preferred model.

\subsubsection{Cosmological Start-Up Process}

Continuing this scenario, the matter fabric obtained the edge in supremacy, and grew that to dominate the emerging cosmos. This fabric then controlled which branch the subsequent remanufacturing process took, and thus antielectrons were converted to protons, rather than electrons to antiprotons. Thus the proto-universe became dominated by matter, rather than antimatter. The cascade of formation-annihilation would have produced neutrino-species, and expelled them outward, thereby creating the fabric. A cloud of photons would have followed, thus reducing the energy available for genesis. Eventually the genesis photon cloud would be too cool and lacking in density, and the formation of matter would abruptly cease.

\subsubsection{Why Do We Not See This Process Today?}

According to this theory, the outward expansion of the universe has reduced the fabric density, to the point where the density in the current epoch is insufficient to convert antielectrons into protons. So the antielectrons from the pair production process are allowed to exist at this stage, whereas in the early universe they would have been converted to protons.

\subsection{Proton Instability}

Looking at the equation $n \times y=>e+p+2 \underline{v}$ and noting that in general all these equations can be rearranged (particules change hand when transferred across the equality), suggests that that the proton may not be absolutely stable. Interacting it with two antineutrinos may remanufacture it as follows:

$$
\begin{aligned}
& p+2 v \Rightarrow p\left(r_{11}{ }^{1} \cdot a_{1} \cdot t_{1}\right)+[\uparrow \uparrow \uparrow]+[\downarrow \downarrow \downarrow]+2 \underline{v} \\
& \Rightarrow p\left(r_{11}{ }^{1} \cdot a_{1} \cdot t_{1}\right)+[2 y]+[2 y]+\underline{v}_{1}\left(r_{\underline{1}} \cdot a \cdot t_{\underline{1}}{ }^{\underline{1}}\right)+\underline{v}_{2}\left(r_{\underline{1}} \cdot a \cdot t_{\underline{1}}{ }^{\underline{1}}\right) \\
& \Rightarrow\left(r_{11}^{1}{ }_{1.1}^{1.1} \cdot a_{1} \cdot t_{1.11} \underline{11}\right)+[4 y] \\
& \Rightarrow \underline{e}\left(r_{\underline{1}} \cdot a_{\underline{1}} \cdot t_{\underline{1}}\right)+\left(r_{11}^{1} \underline{1} \cdot \underline{1} \cdot a_{1}^{\underline{1}} \cdot t_{1}^{\underline{1}}\right)+[4 y] \\
& \Rightarrow \underline{e}+\left(r_{1}^{\underline{1}} \cdot a_{1}{ }^{\underline{1}} \cdot t_{1}^{\underline{1}}\right)+\left(r_{1}^{1}{ }^{1} \cdot a \cdot t\right)+[4 y] \\
& \Rightarrow \underline{e}+(\downarrow \downarrow \downarrow)+(\uparrow \downarrow)+[4 y] \\
& \Rightarrow \underline{e}+2 y+z+[4 y] \\
& \Rightarrow \underline{e}+6 y+z \text {. }
\end{aligned}
$$

What this means is that the proton could unravel back into a positron and photons, with the right kind of inducement by antineutrinos. This prediction may be testable and falsifiable. This result also implies that proton decay would not be fundamentally random, but rather a result of a specific coincidence of antineutrinos. A HED analysis suggests that presupplying the proton with two neutrinos has no effect, i.e. the manufacturing processes are highly asymmetrical. Another possible process is direct decay of the proton:

$$
\begin{aligned}
& p\left(r_{11}{ }^{1} \cdot a_{1} \cdot t_{1}\right)+[\uparrow \uparrow \uparrow]+[\downarrow \downarrow \downarrow] \Rightarrow p\left(r_{11}{ }^{1} \cdot a_{1} \cdot t_{1}\right)+\left[\begin{array}{ccc}
1 & 1 & 1 \\
1 & 1 & 1
\end{array}\right]+\left[\begin{array}{ccc}
1 & 1 & 1 \\
1 & 1 & 1
\end{array}\right] \\
& \Rightarrow v\left(r_{1}^{1} \cdot a \cdot t_{1}^{1}\right)+\underline{e}\left(r_{\underline{1}} \cdot a_{\underline{1}} \cdot t_{1}\right)+v\left(r_{1}^{1} \cdot a \cdot t_{1}^{1}\right)+\left[r_{1}^{\underline{1}} \cdot a_{1}^{1} \cdot t_{1}^{\underline{1}}\right] \\
& \Rightarrow \underline{e}+2 v+2 y \text {. }
\end{aligned}
$$

Thus the proton may spontaneously decay into an antielectron, two neutrinos, and two photons. Decay in the Cordus theory is thought to be initiated by perturbation from the discrete forces of the fabric. In which case the spontaneous proton decay would appear to require a strong antimatter fabric (see next section) to initiate the 
process. This fabric no longer exists, and hence the proton may be relatively stable against this remanufacturing process, in our universe.

\section{Discussion}

\subsection{Summary of the Genesis Production Sequence}

The genesis production sequence, as predicted by the Cordus theory, is shown in Figure 4. This diagram is a high-level representation of the proposed relationships of causality, hence a conceptual formalism, and uses integration definition zero (IDEF0) [44] systems notation. ${ }^{1}$ The genesis production sequence starts with a pair of photons being converted, via pair production, into an electron and antielectron (1). The Cordus theory explains how [40]. The antielectron remanufacturing processes, described here, convert the antielectron into a proton (2). The asymmetry in the manufacturing processes arises from domain warfare between the matter-antimatter species (3). Neutrons are formed by electron capture or beta plus decay (4), for which a Cordus explanation is available [38]. Thus all the components of the atom are accounted for: proton, neutron, and electron. The Cordus theory also explains the strong force, as a synchronization between discrete forces of neighbouring particules [29], and the structure of the atomic nucleus [45]. It also explains the stability trends and drip lines in the table of nuclides ( $\mathrm{H}-\mathrm{Ne})$ [28]. This is much more than other theories, and shows the extent to which the Cordus theory is able to radically reconceptualise the genesis process. So it is conceptually possible to start with the discrete

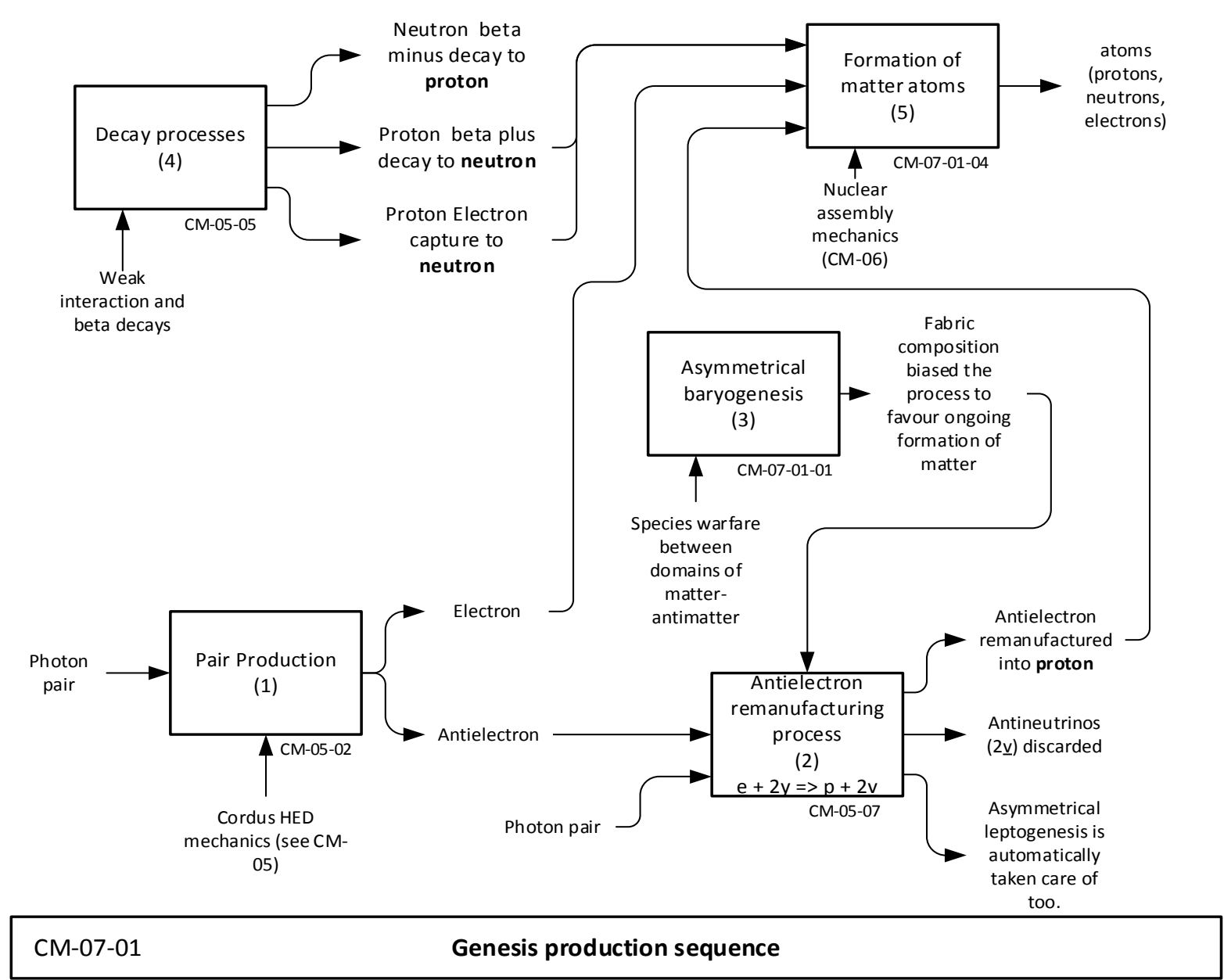

Figure 4. Genesis production sequence. Representation of the overall Cordus theory for the genesis production processes that resulted in matter.

${ }^{1}$ With IDEF0 the object types are inputs, controls, outputs, and mechanisms (ICOM) and are distinguished by placement relative to the box, with inputs always entering on the left, controls above, outputs on the right, and mechanisms below. 
field structures of the photon, i.e. the evanescent field, and explain within one logically consistent theory how these are manufactured into the matter nuclides.

\subsection{Qualitative Summary of Genesis}

This genesis process is therefore conceptually simple: two initial photons are converted into an electron and an antielectron. These radiate photons. The antielectron receives more photons, the field structures of which are used to form a larger structure that re-assembles into a proton and two antineutrinos. The antimatter hand of the antielectron is carried away by the antineutrinos. The remanufacture process initially had two balanced workstreams, converting antielectrons into protons, and electrons into antiprotons. However the process was biased into the matter production stream, perhaps because the two process streams oscillated in their dominance and this was frozen-in as the system cooled.

The original electron and proton combine to form a simple hydrogen atom. The antineutrinos have almost no reactivity with matter, so they simply escape the scene. This is fortunate as the model predicts that antineutrinos can denature a proton. The antineutrinos produced at the original genesis of the universe will now mostly be at the outer edge of the universe, having got into motion before the massy particules. Finally, note that production paths for the neutron are already known in the $\beta$ decays. There is also a Cordus theory that anticipates how these processes work at the level of discrete forces [38].

\subsection{Remanufacturing Processes for Matter}

Taken together, the Cordus theory offers a complete set of forward production processes for electron, proton, and neutron, through to the nuclides. The remanufacturing processes for matter are shown in Figure 5.

\subsection{Resolving the Asymmetry}

The significance is that we do not need to worry about the asymmetry of baryogenesis. Where has all the antimatter gone? Hiding in plain sight, having been remanufactured into the matter baryons themselves. ${ }^{2}$ This is also a parsimonious solution as not only does it explain asymmetrical baryogenesis, but asymmetrical leptogenesis is automatically taken care of too, since the antielectron is consumed in the process.

\subsection{What Has Been Achieved?}

This work makes several novel contributions of a conceptual nature. The first is the identification of a process for remanufacturing an energetic antielectron into a proton and two antineutrinos. The idea itself is a novel contribution, which has not previously been fielded. Another novel contribution is the HED mechanics, with which it has been possible to determine a plausible set of remanufacture mechanics. A third contribution is the identification of the functionality of the neutrino-species. They are found to remove matter-antimatter species hand, and therefore have a crucial role in the genesis production sequence. While neutrinos have featured in other baryogenesis scenarios, they have not had any role like this. In addition, the proposed production process itself is detailed, and the inputs and outputs are predicted, which makes it potentially testable and falsifiable.

A fifth contribution is that this process accounts for both asymmetrical baryogenesis as well as leptogenesis. This is a parsimonious solution: by comparison other theories need additional mechanisms for the two asymmetries. A sixth contribution is the identification of frozen domain warfare being a possible deeper mechanism for the asymmetry. This is a conceptually simple and efficient explanation, and obviates the need for elaborate mechanisms or coincidences. A seventh contribution is identification of the conditions under which the proton may decay. While the idea of proton decay is not new, the prediction that it is susceptible to impact by two antineutrinos is a novel contribution and one that also may be tested and falsified.

Another novel contribution is the breadth of the theory. It explains the whole process from the mass-energy equivalence of pair production, through to the preponderance of matter over antimatter at genesis, and onward to nucleosynthesis of the nuclides and their decay processes. All these process can be represented in part by other theories, but not in an integrated solution for the whole process.

\footnotetext{
${ }^{2}$ This explanation suggests that it could be true, in a way, to say that the antimatter has been pushed to another part of the universe. However it is not antimatter in the form of antiatoms, antisuns, and antigalaxies, but a plain desert of relatively inert antineutrinos spread through the matter universe and concentrated at its horizon.
} 


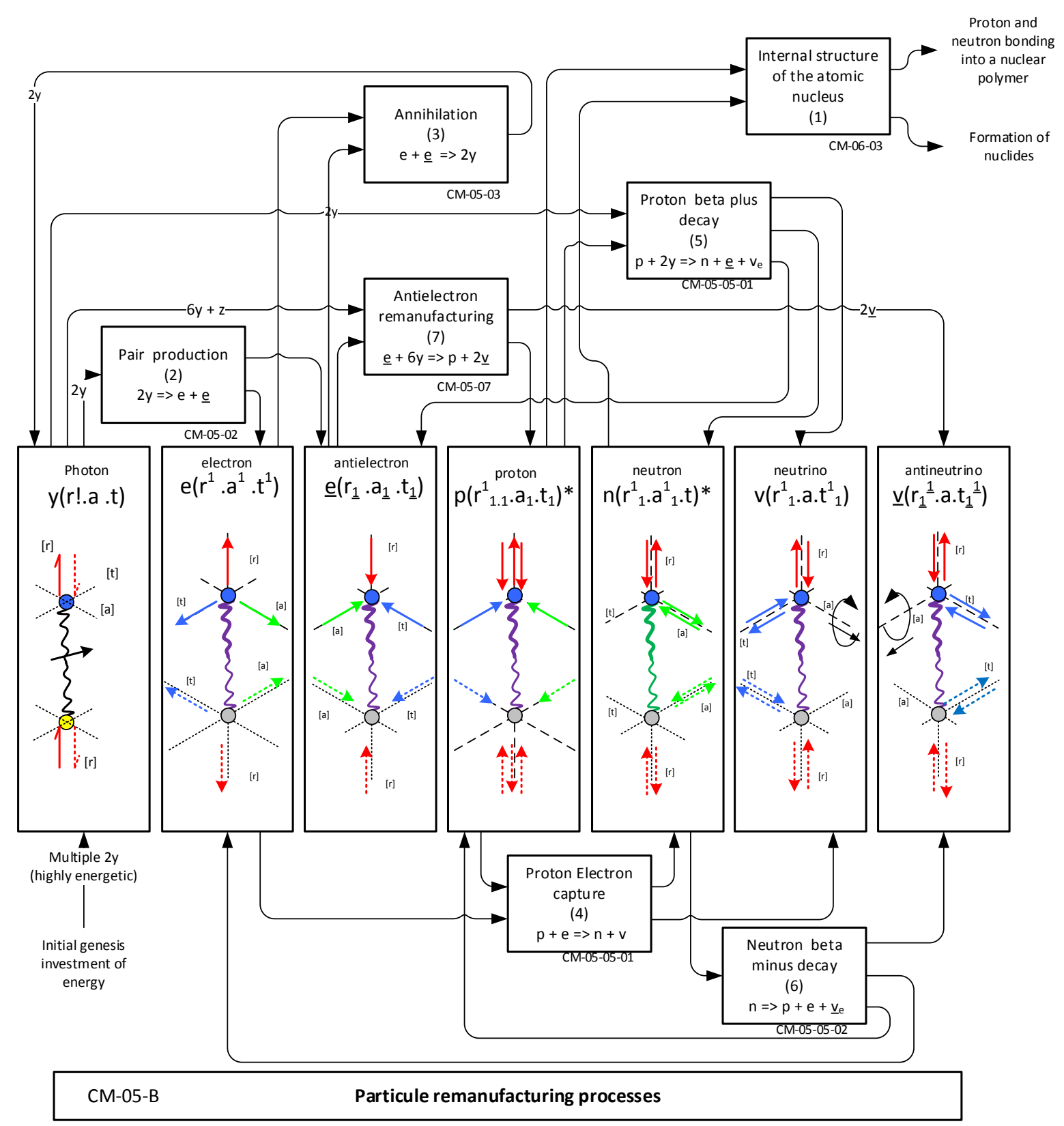

Figure 5. Remanufacturing processes for matter. Summary of the remanufacturing routes for the genesis production sequence for a matter universe. This diagram shows the proposed discrete force structures for a variety of particules.

\subsection{Implications}

The implications, if this theory is correct, is that the matter-antimatter asymmetry of genesis is explainable as the remanufacture of the antielectron into the proton. Importantly, this does not require any new particles or new forces, unlike most of the competing theories. All it requires is a plausible set of assumptions in a covert-structure design. Another implication of this theory is that the asymmetries in the baryogenesis and leptogenesis processes are conjoined. This is in contrast to the conventional perspective that treats them as independent.

The corollary is that the Cordus theory is shown to be capable of profoundly novel solutions for both fundamental physics and cosmology. It is relevant to note that the Cordus theory has achieved not only a solution for asymmetrical genesis, as shown here, but also a comprehensive and logically consistent set of explanations for a wide variety of physical phenomena, including wave-particle duality, unification of forces, annihilation, decay, 
the nuclides ( $\mathrm{H}$ to $\mathrm{Ne}$ ), among many other phenomena. All those are ontologically problematic for conventional physics. If the theory is true, then a radical revision is necessary in foundational physics, the deeper reality is deterministic after all [27], locality fails, particles have inner structure, and quantum mechanics is re-interpreted as a stochastic approximation of a deeper and finer mechanics. These audacious implications arise from a critique of the 0 -D premise.

Taken together with other developments, the Cordus theory offers a complete set of forward production processes from the energy of the evanescent field of the photon [40], through genesis [this paper] and the strong force (synchronous interaction) [29], up to the nuclides [28], back down through the decay processes [38], to annihilation back to energy [41] [42]. The processes for mass-energy equivalence are therefore fully mapped out at the conceptual level. In contrast to other competing theories of genesis, this only requires particles already known to the Standard Model. However it does require them to have a specific covert-structure. The theory shows that if particles were to have such a structure then plausible solutions arise for asymmetrical genesis. This does not imply that all NLHV theories are so endowed.

\subsection{Implications for Future Work}

While the theory has high coherence (internal validity), and provides an excellent fit to empirical data (e.g. for the nuclides [28]), it is conceptual in nature. Consequently a mathematical formalism is not yet available for the theory. This is an area for future development as the theory matures. There is reason to believe that it should be possible to express the Cordus theory in such a formalism, but this is a significant venture that is beyond the scope of this paper, and is instead left to future work. It might be worth starting a formalism using string theory, due to the dimensional similarity of these theories. QM is not expected to be a suitable starting point as it is premised on 0-D point particles which are simplifications of the Cordus particule. Second, there is more conceptual work to be done to explain other aspects of cosmology and the nucleosynthesis of the heavier elements. There are deeper foundational questions to explore too: how the reactive ends transform, and the composition of the fibrils and discrete forces. At this point we simply propose their existence as necessary for the Cordus model, and leave their elucidation for future work.

\section{Conclusions}

What has been achieved here is a novel alternative conceptual theory for the asymmetry of matter over antimatter in the universe. The development started with the basic Cordus proposition that particles are not 0-D points but have a distinct internal structure with two ends and discrete external fields. This leads to a theory for electron-antielectron pair-production, showing how the structures of the photon can be reassembled into an electron and antielectron [40]. The present paper demonstrates the conceptually feasible of the antielectron being remanufactured into a proton, discarding antineutrinos in the process. A specific process has been predicted for this. The original electron and proton combine to form a simple hydrogen atom. The antineutrinos have little reactivity, so they escape. The antimatter field structure of the antielectron is carried away by the antineutrinos as a waste stream. An explanation is provided for why the matter hand prevailed over antimatter during the cosmological start-up process.

To answer the question identified at the outset: why is there more matter than antimatter in the universe? Our answer is that the initial genesis process converted energy into equal quantities of matter and antimatter, in the form of electrons and antielectrons (positrons). It is proposed that a second process converted the antielectrons into the protons, and the waste antimatter component was carried off by antineutrinos. Therefore according to this interpretation, the apparent asymmetry of baryogenesis is because the antimatter is hiding in plain sight, having been remanufactured into the matter baryons themselves.

\section{Author Contributions}

All authors contributed to the creation of the underlying concept, development of the ideas, and editing of the paper. DP created the drawings.

\section{Conflict of Interest Statement}

The authors declare that there is no conflict of interests regarding the publication of this article. The research 
was conducted without personal financial benefit from any third party funding body, nor did any such body influence the execution of the work.

\section{References}

[1] Riotto, A. (2011) Journal of Physics: Conference Series, 335, Article ID: 012008.

[2] Riotto, A. and Trodden, M. (1999) Annual Review of Nuclear and Particle Science, 49, 35-75. http://dx.doi.org/10.1146/annurev.nucl.49.1.35

[3] Sakharov, A.D. (1967) Journal of Experimental and Theoretical Physics Letters, 5, 24-27.

[4] Dine, M. and Kusenko, A. (2003) Reviews of Modern Physics, 76, 1-30. http://dx.doi.org/10.1103/RevModPhys.76.1

[5] Dolgov, A. (2000) Matter-Antimatter Domains in the Universe. In: Frontiers in Particle Astrophysics and Cosmology, Elsevier, Netherlands.

[6] Flanz, M., Paschos, E.A. and Wu, Y.L. (1993) Physics Letters B, 315, 379-385. http://dx.doi.org/10.1016/0370-2693(93)91628-Z

[7] Lambiase, G., Mohanty, S. and Prasanna, A.R. (2013) International Journal of Modern Physics D, 22.

[8] Alexander, S.H.S., Peskin, M.E. and Sheikh-Jabbari, M.M. (2006) Physical Review Letters, 96, Article ID: 081301.

[9] Di Bari, P. (2012) Contemporary Physics, 53, 315-338. http://dx.doi.org/10.1080/00107514.2012.701096

[10] Ho, C.M. (2011) Physics Letters B, 702, 398-401. http://dx.doi.org/10.1016/j.physletb.2011.07.035

[11] Gagnon, J.S. and Shaposhnikov, M. (2011) Physical Review D, 83, Article ID: 065021. http://dx.doi.org/10.1103/PhysRevD.83.065021

[12] Fong, C.S., Nardi, E. and Riotto, A. (2012) Advances in High Energy Physics, 2012, Article ID: 158303. http://dx.doi.org/10.1155/2012/158303.

[13] Trodden, M. (1999) Reviews of Modern Physics, 71, 1463-1500. http://dx.doi.org/10.1103/RevModPhys.71.1463.

[14] Schmidt, M.G. (2011) Progress in Particle and Nuclear Physics, 66, 249-253. http://www.sciencedirect.com/science/article/pii/S0146641011000159

[15] Gleiser, M. and Kolb, E.W. (1992) Physical Review Letters, 69, 1304-1307. http://dx.doi.org/10.1103/PhysRevLett.69.1304

[16] Klinkhamer, F.R. (1985) Zeitschrift für Physik C Particles and Fields, 29, 153-160. http://dx.doi.org/10.1007/bf01571398.

[17] Affleck, I. and Dine, M. (1985) Nuclear Physics B, 249, 361-380. http://www.sciencedirect.com/science/article/pii/0550321385900215

[18] Buchmuller, W., Schmitz, K. and Vertongen, G. (2011) Nuclear Physics B, 851, 481-532. http://dx.doi.org/10.1016/j.nuclphysb.2011.06.004.

[19] Flanz, M., Paschos, E.A. and Sarkar, U. (1995) Physics Letters B, 345, 248-252. http://dx.doi.org/10.1016/0370-2693(94)01555-q.

[20] Flanz, M., Paschos, E.A., Sarkar, U. and Weiss, J. (1996) Physics Letters B, 389, 693-699. http://dx.doi.org/10.1016/s0370-2693(96)01337-8

[21] Bechtle, P., Sarrazin, B., Desch, K., Dreiner, H.K., Wienemann, P., Kramer, M., Robens, C. and O’Leary, B. (2011) Physical Review D, 84, Article ID: 011701, 5 p. http://dx.doi.org/10.1103/PhysRevD.84.011701.

[22] Dutta, B. and Kumar, J. (2011) Physics Letters B, 699, 364-367. http://dx.doi.org/10.1016/j.physletb.2011.04.036.

[23] Haba, N. and Matsumoto, S. (2011) Progress of Theoretical Physics, 125, 1311-1316. http://dx.doi.org/10.1143/ptp.125.1311.

[24] Kamada, K. and Yamaguchi, M. (2012) Physical Review D, 85, Article ID: 103530, 14 p. http://dx.doi.org/10.1103/PhysRevD.85.103530.

[25] Falkowski, A., Ruderman, J.T. and Volansky, T. (2011) Journal of High Energy Physics, 106. http://dx.doi.org/10.1007/jhep05(2011)106.

[26] Pons, D.J., Pons, A.D., Pons, A.M. and Pons, A.J. (2012) Physics Essays, 25, 132-140.

[27] Einstein, A., Podolsky, B. and Rosen, N. (1935) Physical Review, 47, 777. http://link.aps.org/doi/10.1103/PhysRev.47.777

[28] Pons, D.J., Pons, A.D. and Pons, A.J. (2013) Applied Physics Research, 5, 145-174. http://dx.doi.org/10.5539/apr.v5n6p145 
[29] Pons, D.J., Pons, A.D. and Pons, A.J. (2013) Applied Physics Research, 5, 107-126. http://dx.doi.org/10.5539/apr.v5n5107

[30] Bell, J.S. (1964) Physics, 1, 195-200. http://philoscience.unibe.ch/documents/TexteHS10/bell1964epr.pdf

[31] Leggett, A. (2003) Foundations of Physics, 33, 1469-1493. http://dx.doi.org/10.1023/a:1026096313729

[32] Groblacher, S., Paterek, T., Kaltenbaek, R., Brukner, C., Zukowski, M., Aspelmeyer, M. and Zeilinger, A. (2007) Nature, 446, 871-875. http://dx.doi.org/10.1038/nature05677

[33] Laudisa, F. (2008) Foundations of Physics, 38, 1110-1132. http://dx.doi.org/10.1007/s10701-008-9255-8

[34] De Zela, F. (2008) Journal of Physics A: Mathematical and Theoretical, 41, Article ID: 505301. http://dx.doi.org/10.1088/1751-8113/41/50/505301

[35] de Broglie, L. (1925) Annales de Physique, 3.

[36] Bohm, D. and Bub, J. (1966) Reviews of Modern Physics, 38, 453-469. http://dx.doi.org/10.1103/RevModPhys.38.453

[37] Colbeck, R. and Renner, R. (2011) Nature Communications, 2, 5. http://dx.doi.org/10.1038/ncomms1416

[38] Pons, D.J., Pons, A.D. and Pons, A.J. (2014) Applied Physics Research, 6, 50-63. http://dx.doi.org/10.5539/apr.v6n3p50

[39] Pons, D.J., Pons, A.D. and Pons, A.J. (2013) Applied Physics Research, 5, 23-47. http://dx.doi.org/10.5539/apr.v5n6p23

[40] Pons, D.J., Pons, A.D. and Pons, A.J. (2014) Nuclear and Atomic Physics. viXra:1404.0051

[41] Pons, D.J., Pons, A.D. and Pons, A.J. (2014) Physics Essays, 27, 26-35.

[42] Pons, D.J., Pons, A.D. and Pons, A.J. (2014) Applied Physics Research, 6, 28-46. http://dx.doi.org/10.5539/apr.v6n2p28

[43] Pons, D.J. and Pons, A.D. (2013) The Open Astronomy Journal, 6, 77-89. http://dx.doi.org/10.2174/1874381101306010077

[44] FIPS (1993) Integration Definition for Function Modeling (IDEF0). http://www.itl.nist.gov/fipspubs/idef02.doc

[45] Pons, D.J., Pons, A.D. and Pons, A.J. (2013) Nuclear and Atomic Physics. viXra:1309.0010 
Scientific Research Publishing (SCIRP) is one of the largest Open Access journal publishers. It is currently publishing more than 200 open access, online, peer-reviewed journals covering a wide range of academic disciplines. SCIRP serves the worldwide academic communities and contributes to the progress and application of science with its publication.

Other selected journals from SCIRP are listed as below. Submit your manuscript to us via either submit@scirp.org or Online Submission Portal.
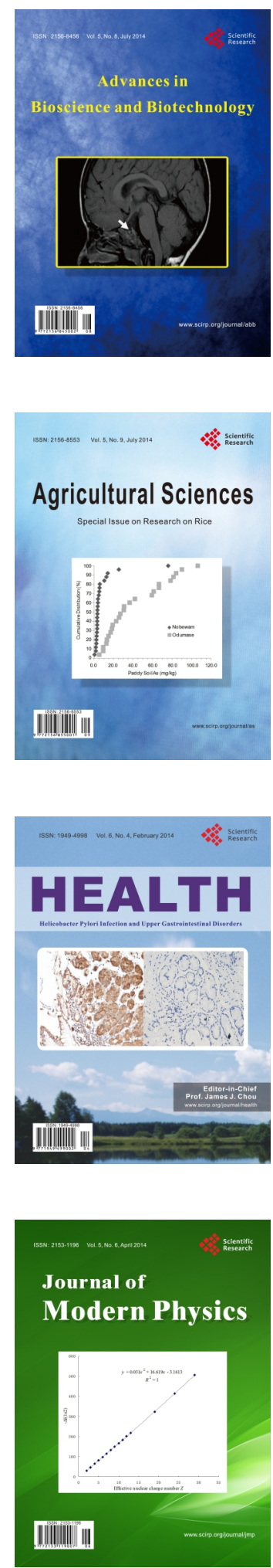
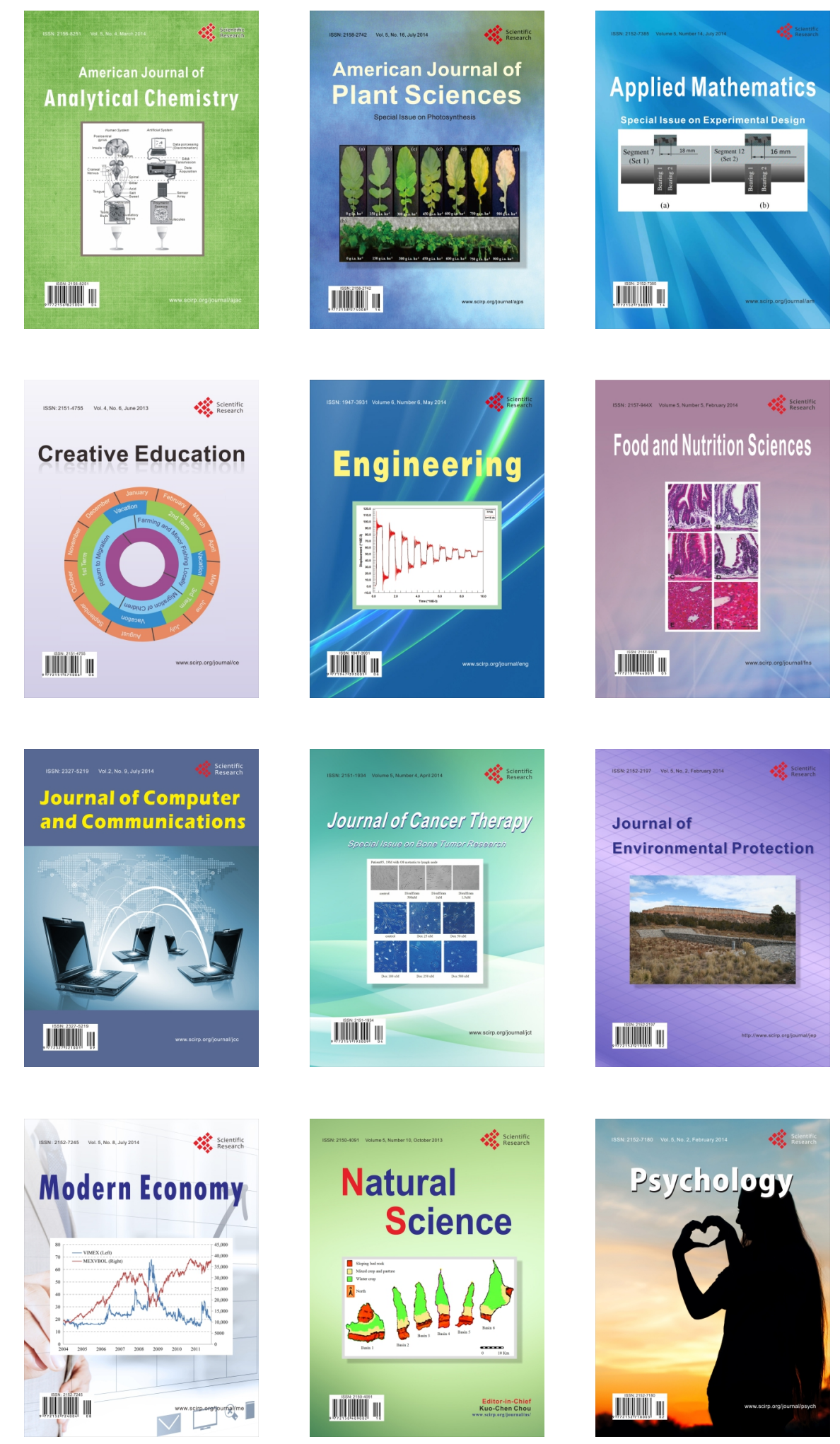